\title{
Akt-dependent activation of Erk by cyclin D1b contributes to cell invasiveness and tumorigenicity
}

\author{
CHUL JANG KIM $^{1}$, YUKIHIRO TAMBE ${ }^{2}$, KEN-ICHI MUKAISHO ${ }^{3}$, \\ HIROYUKI SUGIHARA ${ }^{3}$, AKIHIRO KAWAUCHI ${ }^{4}$ and HIROKAZU INOUE ${ }^{2}$ \\ ${ }^{1}$ Department of Urology, Kohka Public Hospital, Kohka, Shiga 528-0014; \\ Divisions of ${ }^{2}$ Microbiology and Infectious Diseases and ${ }^{3}$ Molecular and Diagnostic Pathology; \\ ${ }^{4}$ Department of Urology, Shiga University of Medical Science, Otsu, Shiga 520-2192, Japan
}

Received May 9, 2015; Accepted September 27, 2016

DOI: $10.3892 / 01.2016 .5286$

\begin{abstract}
A total of two major isoforms, cyclin D1a and cyclin D1b, are generated from the human cyclin D1 gene by alternative splicing. Cyclin D1b is scarcely expressed in normal tissues; however, it is expressed at a high frequency in certain types of cancerous tissue. The present authors previously constructed cyclin D1b transgenic $(\mathrm{Tg})$ mice and identified rectal tumors, including adenocarcinoma and sessile serrated adenoma, in $62.5 \%$ of female $\mathrm{Tg}$ mice. In addition, the present authors indicated that cyclin D1b expression enhances phosphorylation of extracellular signal-regulated kinase (Erk) in these rectal tumors, and in mouse embryonic fibroblast (MEF) cells and human 293T cells. In the present study, it was initially demonstrated that cyclin D1b has the ability to enhance cell invasiveness by itself; it additionally increases cell invasiveness, anchorage-independent growth and tumorigenicity in cooperation with an activated $K$-ras oncogene in MEF cells. Phosphorylation of Akt was increased in cyclin D1b-expressing MEF cells and in the rectal tumor tissues of cyclin D1b Tg mice. Phosphorylation of Akt was also enhanced by transfection of cyclin D1b, but not cyclin D1a, in human 293 T cells. Treatment with an Akt inhibitor suppressed phosphorylation of Erk in 293T cells expressing cyclin D1b and D1bTgRT cells established from rectal cancer of the cyclin D1b Tg mouse. Furthermore, the Akt inhibitor suppressed the invasiveness of D1bTgRT cells and the tumor growth of these cells in nude mice when the Akt inhibitor was injected into the tumors. These results indicate that cyclin D1b activates Erk through Akt, and that activation of Akt contributes to the tumorigenicity of the cyclin D1b Tg mice. Inhibitors targeting the phosphoinositide 3-kinase/Akt signaling pathway are thus
\end{abstract}

Correspondence to: Dr Hirokazu Inoue, Division of Microbiology and Infectious Diseases, Shiga University of Medical Science, Setatsukinowa-cho, Otsu, Shiga 520-2192, Japan

E-mail: hirokazu@belle.shiga-med.ac.jp

Key words: cyclin D1b, Akt, extracellular signal-regulated kinase, rectal tumorigenesis expected to have therapeutic potential in a variety of human cancer types expressing cyclin D1b.

\section{Introduction}

A total of two major isoforms of human cyclin D1 are generated via alternative splicing: cyclin D1a and cyclin D1b (1). Cyclin D1a mRNA has a coding region of $882 \mathrm{bp}$ within five exons (1). The cyclin D1b mRNA lacks exon 5 but has an extended exon 4 due to nonsplicing; within intron 4 , there is a translation stop codon (1). The cyclin Dlb mRNA encodes a 275-amino acid protein. It differs at the C-terminus from the 294-amino acid protein encoded by cyclin Dla mRNA (1). Cyclin Dla is expressed ubiquitously and has been shown to control the G1-S transition of the cell cycle $(2,3)$. It binds to cyclin-dependent kinase (CDK) $4 / 6$ to phosphorylate the retinoblastoma $(\mathrm{Rb})$ protein $(2,3)$. Although expression of cyclin Dlb is barely detectable in normal tissue, it has been detected in various types of cancer, including bladder cancer, esophageal cancer, colon cancer, B-lymphoid malignancies, breast cancer, and prostate cancer, Ewing's sarcoma and mantle cell lymphoma (4). Gene transfer experiments have indicated that cyclin D1b is more oncogenic than cyclin Dla (5-8).

A total of two distinct mRNA transcripts, isoform $a$ and $b$, were produced by a polymorphism, A870G, located at the splice donor region in the exon-intron 4 boundary $(9,10)$. The G870 allele generates the well-described cyclin D1a transcript $(9,10)$. An alternative spliced product, cyclin D1b, results from the A870 allele. This allele hinders splicing and allows for read through into intron 4 and a premature termination of transcription $(9,10)$. As individuals who are homozygous for $\mathrm{G} / \mathrm{G}$ continue to produce a cyclin D1b transcript $(9,11)$, it is difficult to explain the production of the cyclin D1b transcript by the mechanism of polymorphism. Recently, Paronetto et al (12) showed that the RNA-binding protein Sam68 regulates alternative splicing of cyclin D1.

Previously, it has been demonstrated that ectopic expression of cyclin D1b promotes cell invasiveness and anchorage-independent growth in human bladder cancer cells (13). However, the introduced cyclin D1b was not able to associate with CDK4 and enhance $\mathrm{Rb}$ phosphorylation (13), showing that the function of cyclin $\mathrm{D} 1 \mathrm{~b}$ is independent of $\mathrm{Rb}$ phosphorylation in the 
enhancement of cell invasiveness and anchorage-independent growth.

The present authors previously constructed cyclin D1b transgenic ( $\mathrm{Tg}$ ) mice to clarify the in vivo oncogenic potential of cyclin D1b and observed that rectal tumors developed in $62.5 \%$ of the female Tg mice (14). All rectal tumors in cyclin D1b Tg mice revealed histological characteristics similar to human sessile serrated adenoma/polyps (SSA/Ps) (15-20). Adenocarcinomas were also detected in $53 \%$ of these rectal tumors (14). This suggested that these adenocarcinomas arose from the SSA/P-like lesions. No rectal tumors developed in the ovariectomized female cyclin D1b Tg mice, showing that ovarian hormones are critical for rectal carcinogenesis in these Tg mice (14). Phosphorylation of extracellular signal-regulated kinase (Erk), without activation of mitogen-activated protein kinase (MAPK)/Erk kinase (MEK), and expression of estrogen receptor (ER)- $\beta$ were increased in the rectal tumors of female cyclin D1b Tg mice in comparison with normal rectums of female wild-type (WT) mice. Activation of Erk was also observed in mouse embryo fibroblast (MEF) cells ectopically expressing cyclin D1b. Furthermore, a tumor cell line, D1bTgRT, was established from a rectal cancer of a female cyclin D1b Tg mouse (14). Knockdown of cyclin D1b by small interfering (si)RNA in this cell line suppressed phosphorylation of Erk, anchorage-independent growth, cell invasiveness and tumorigenicity in nude mice. These results demonstrate that the expression of cyclin D1b has a significant role in female-specific rectal carcinogenesis via Erk activation and expression of ER- $\beta$ in this mouse model.

In the present study, the effects of cyclin D1b on cell transformation and the mechanism of Erk activation independent of MEK activation in MEF, 293T and D1bTgRT cells were examined and it was observed that cyclin D1b activates Erk through Akt. In addition, the present study investigated the role of Akt activation in the rectal tumorigenesis of cyclin D1b Tg mice and showed that enhanced phosphorylation of Akt by cyclin Dlb contributes to rectal tumorigenicity.

\section{Materials and methods}

Expression plasmids. The cloning of cyclin D1b complementary DNA and construction of the expression plasmids, pCR3.1-cyclin D1b-Flag and pCR3.1-cyclin D1a-Flag, and pCR3.1-cyclin D1-T286A-Flag were performed as previously described (13).

Cell culture. A tumor cell line, D1bTgRT, was established from a rectal tumor of a cyclin D1b Tg mouse. D1bTgRT cells were cultured in RPMI-1640 medium (Nacalai Tesque, Inc., Kyoto, Japan) supplemented with $10 \%$ fetal calf serum (FCS) (Sigma-Aldrich; Merck Millipore, Darmstadt, Germany), penicillin $(100 \mathrm{U} / \mathrm{ml})$ and streptomycin $(100 \mu \mathrm{g} / \mathrm{ml})$ in humidified air containing $5 \% \mathrm{CO}_{2}$ at $37^{\circ} \mathrm{C}$. MEF cells prepared from the embryos of WT and cyclin D1b Tg mice were immortalized by the large $\mathrm{T}$ antigen of Simian vacuolating virus 40 (SV40) (14). Furthermore, MEF cells were infected with retrovirus expressing activated $K$-ras, known as pBABEpuro-K-Ras (21). 293T cells derived from human embryonic kidney were used for plasmid transfection. These cells were cultured in Dulbecco's modified Eagle's medium
(DMEM) (Nacalai Tesque, Inc.) supplemented with $10 \%$ FCS, penicillin $(100 \mathrm{U} / \mathrm{ml})$ and streptomycin $(100 \mu \mathrm{g} / \mathrm{ml})$ in humidified air containing $5 \% \mathrm{CO}_{2}$ at $37^{\circ} \mathrm{C}$.

Cell proliferation assay. Cell proliferation assay was performed by culturing the cells in $35-\mathrm{mm}$ culture dishes. A total of $2 \times 10^{4}$ of the cells were cultured in triplicate into each dish and incubated at $37^{\circ} \mathrm{C}$. Viable cells were trypsinized and counted using a hemocytometer.

In vitro invasion assay. The in vitro cell invasiveness was evaluated by a Matrigel ${ }^{\mathrm{TM}}$ Basement Membrane Matrix Invasion Chamber (chamber size, $6.4 \mathrm{~mm}$; membrane surface area, $0.3 \mathrm{~cm}^{2}$; pore size, $8 \mu \mathrm{m}$; BD Biosciences, Franklin Lakes, NJ, USA), according to the manufacturer's protocol (13). A total of $500 \mu \mathrm{l}$ of cell suspension $\left(5 \times 10^{4}\right.$ cells $\left./ \mathrm{ml}\right)$ was added to each chamber. The chambers containing the cells were incubated for 4 days in a $\mathrm{CO}_{2}(5 \%)$ incubator. Noninvasive cells were removed from the upper surface of the membrane. The invasive cells on the underside were stained with Diff-Quik ${ }^{\mathrm{TM}}$ stain (Kokusai-Shiyaku, Kobe, Japan) and counted under a microscope (TMS; Nikon Corporation, Tokyo, Japan). Each cell sample was evaluated in triplicate.

Soft agar assay. Anchorage-independent growth of the cells was evaluated by colony-forming ability in soft agar (13). A total of 10,000 cells were inoculated into a $60-\mathrm{mm}$ dish containing $0.4 \%$ Noble agar containing DMEM supplemented with $10 \% \mathrm{FCS}$ and incubated at $37^{\circ} \mathrm{C}$. The number of colonies $(>0.15 \mathrm{~mm}$ in diameter) on each plate was scored after 3 weeks of incubation. Each assay was performed in triplicate.

Tumorigenicity in nude mice. Tumorigenicity of the cells was examined by subcutaneous injection of $3 \times 10^{6}$ cells per $0.2 \mathrm{ml}$ PBS (-) into 6-week-old BALB/c-nu/nu female nude mice $(20 \mathrm{~g})$. The animals were housed in a specific pathogen-free room with controlled temperature $\left(20-22^{\circ} \mathrm{C}\right)$, humidity (50-60\%) and a pre-set light-dark cycle (12:12 h). They were allowed ad libitum access to food (CE-2; CLEA Japan, Inc., Tokyo, Japan) and water. A total of four weeks subsequent to injection, the tumorigenic potential of the cells was assessed by measuring the weight and volume of tumors. The use of the animals in the experimental protocols was reviewed and approved by the Committee of Research Center for Animal Life Science at Shiga University of Medical Science (Otsu, Japan; approval no. 2014-12-5).

Transfection. Transfection of plasmid DNA was performed using Lipofectamine ${ }^{\mathrm{TM}}$ PLUS reagent (Invitrogen; Thermo Fisher Scientific, Inc., Waltham, MA, USA), according to the manufacturer's protocol. Briefly, $5 \times 10^{5}$ cells were inoculated into a $60-\mathrm{mm}$ dish and, upon overnight incubation at $37^{\circ} \mathrm{C}$, $2 \mu \mathrm{g}$ of plasmid DNA was transfected.

Immunoblotting. Lysates of cells and tissues were prepared by Laemmli-sodium dodecyl sulfate (SDS) buffer containing $62.5 \mathrm{mM}$ Tris-HCl (pH 6.8), 10\% glycerol, 5\% 2-mercaptoethanol, $2 \%$ SDS, $0.01 \%$ bromophenol blue and $5 \mathrm{mM}$ ethylenediaminetetraacetic acid. Each cell lysate $(20 \mu \mathrm{g}$ of protein) underwent 10 or $12 \%$ SDS-PAGE, and the proteins 
were electrotransferred to Immobilon-P membranes (EMD Millipore). Following blocking with TBS-T $[10 \mathrm{mM}$ Tris-HCl ( $\mathrm{pH}$ 7.6), $150 \mathrm{mM}$ sodium chloride and $0.1 \%$ Tween-20] containing 5\% bovine serum albumin (BSA) (Nacalai Tesque, Inc.), the membranes were incubated with the primary antibodies diluted 1:1,000 in TBS-T containing 2\% BSA. Following washes with TBS-T, the membranes were incubated for $1 \mathrm{~h}$ at room temperature in horseradish peroxidase-conjugated anti-mouse or or anti-rabbit immunoglobulin G (NA931 and NA934, respectively; GE Healthcare Bio-Sciences, Pittsburgh, PA, USA) diluted 1:20,000 in TBS-T containing $2 \%$ BSA. Following washing with TBS-T, immunoreactivity was detected by an enhanced chemiluminescence system (GE Healthcare Bio-Sciences) using X-ray films.

Antibodies. The primary antibodies used for immunoblotting were as follows: Anti- $\alpha$-tubulin (DM1A) (T9026) and anti-FLAG (M2) (F3165) monoclonal antibodies were obtained from Sigma-Aldrich (Merck Millipore). Anti-phospho Akt (T308 and S473, and D25E6 and D9E) (\#13038 and \#4060, respectively) rabbit monoclonal, anti-Akt rabbit polyclonal (\#9272), anti-phospho Erk1/2 (p44/42 MAPK) rabbit monoclonal (T202/Y204, D13.14.4E) (\#9101), anti-Erk1/2 (p44/42 MAPK) rabbit monoclonal (137F5) (\#4695), anti-phospho-MEK1/2 rabbit monoclonal (S221, 166F8) (\#16211), anti-MEK1/2 rabbit monoclonal (47F6) (\#9126) and anti-cyclin D1 rabbit monoclonal (92G2) (\#2978) primary antibodies, which were purchased from Cell Signaling Technology, Inc. (Danvers, MA, USA). Anti-E-cadherin (\#610181) and anti-N-cadherin (\#13116) monoclonal primary antibodies were purchased from BD Biosciences, and the anti-vimentin (C9) (sc-6260) mouse monoclonal antibody was obtained from Santa Cruz Biotechnology, Inc. (Dallas, TX, USA). All antibodies were used at a dilution of 1:1,000.

In vivo evaluation of tumor growth in nude mice treated by Akt inhibitor injection into tumors. The use of the animals in the experimental protocols was reviewed and approved by the Ethics Committee of the Research Center for Animal Life Science at Shiga University of Medical Science (Otsu, Japan). The right dorsal flank of each 6-week-old $\mathrm{BALB} / \mathrm{c}-\mathrm{nu} / \mathrm{nu}$ female nude mouse was injected subcutaneously with $3 \times 10^{6}$ D 1 bTgRT cells. Following, establishment of palpable tumors $\left(>100 \mathrm{~mm}^{3}\right)$, external tumor volume was determined on days 0,7 and 11 . A total of six mice carrying palpable tumors were randomized into two groups of three mice each. Each Akt inhibitor at a dose of $25 \mathrm{pmol} / \mathrm{mg}$ (calculated tumor weight) or a similar volume of dimethyl sulfoxide (DMSO) was injected into the tumor of each mouse on days 0 and 7. Tumor volume was calculated using the formula: $\mathrm{AxBxC} / 2$, where $\mathrm{A}, \mathrm{B}$ and $\mathrm{C}$ represent the diameters of length, height and width, respectively. The volume of injection for a tumor was prepared as $100 \mu \mathrm{l}$.

Statistical analyses. Using the paired Student's t-test, the present study compared the invasiveness of DlbTgRT cells treated with Akt inhibitor with those treated with DMSO as a control, and the in vivo tumorigenicity of DlbTgRT cells treated with Akt inhibitor with those treated with DMSO as a control, in the same nude mouse. $\mathrm{P} \leq 0.05$ was considered to indicate a statistically significant difference. All statistical analyses were performed using the R statistical software package, version 2.6.2 (The R Project for Statistical Computing, Vienna, Austria).

\section{Results}

Activation of Akt in MEF cells expressing cyclin Dlb. To investigate the role of cyclin D1b in cell transformation and the signaling pathway resulting in Erk activation, the present study established WT and cyclin D1b-expressing MEF cell lines, named WT-LT and D1b-LT, respectively, by introducing the large T antigen of SV40 into embryonic fibroblasts derived from WT and cyclin D1b-Tg mouse. Significant differences were not observed between WT-LT and Dlb-LT cells in terms of cell proliferation (Fig. 1A and B). However, D1b-LT cells had a slightly more spindle-shaped appearance compared with WT-LT cells. Subsequently, to investigate whether the expression of cyclin D1b elevates the sensitivity to in vitro cell transformation, an activated $K$-ras oncogene was introduced into the cell lines using the retrovirus vector pBABE-puro and established vector and $K$-ras-expressing WT-LT and D1b-LT cell lines, named WT-LT/BP, WT-LT/K-ras, D1b-LT/BP and D1b-LT/K-ras. The morphology of D1b-LT/K-ras cells was significantly altered, although the morphology of the WT-LT/K-ras cells was also transformed into a more spindle-like appearance, as compared with D1b-LT cells (Fig. 1A). The proliferation of Dlb-LT/K-ras cells was increased compared with any of the other cells (Fig. 1B). The present study subsequently investigated the anchorage-independent growth of these cells; as shown in Fig. 1C, D1b-LT/K-ras cells produced a large number of colonies in soft agar, whereas WT-LT/BP cells scarcely produced any colonies and WT-LT/K-ras and D1b-LT/BP cells slightly produced colonies. The present study also investigated the tumorigenicity of these cells in nude mice. As shown in Fig. 1D, although WT-LT/K-ras and D1b-LT/K-ras cells demonstrated tumorigenicity in nude mice, the tumor volumes of the D1b-LT/K-ras cells were significantly increased compared with that of the WT-LT/K-ras cells $(\mathrm{P}=0.039)$. Neither WT-LT/BP nor D1b-LT/BP cells produced tumors in nude mice. WT-LT/K-ras and D1b-LT/BP cells showed a more invasive phenotype compared with WT-LT cells in the Matrigel assay, and expression of cyclin D1b and $K$-ras synergistically enhanced cell invasiveness (Fig. 1E). These results indicate that cyclin D1b by itself has weak transformation activity, and cyclin Dlb-expressing MEF cells are more sensitive to transformation by the activated $K$-ras oncogene than WT MEF cells, suggesting that cyclin D1b cooperates with activated $K$-ras to transform MEF cells.

As cyclin D1b enhanced cell invasiveness, the present study investigated alterations to the expression of proteins involved in the epithelial-to-mesenchymal transition and Akt phosphorylation by immunoblot analyses with specific antibodies. As shown in Fig. 2A, expression of E-cadherin was downregulated in WT-LT/K-ras and D1b-LT/BP cells compared with that in WT-LT/BP cells; its expression was completely lost in D1b-LT/K-ras cells. This result is compatible with the morphological alterations observed in transformed MEF cells (Fig. 1A). The levels of $\mathrm{N}$-cadherin and vimentin were similar in all cell lines. Phosphorylation of Akt (T308 and S473 
A

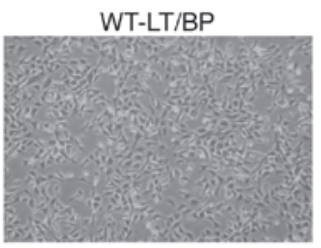

D1b-LT/BP

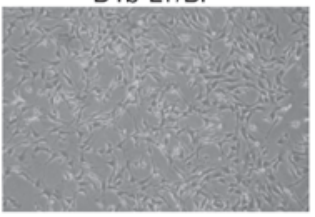

WT-LT/K-ras

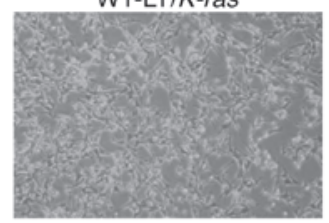

D1b-LT/K-ras

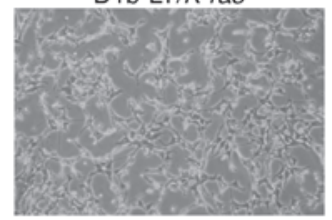

D

C

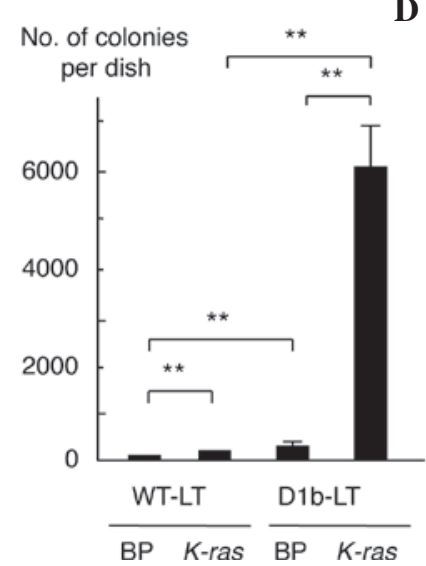

B No. of cells per dish

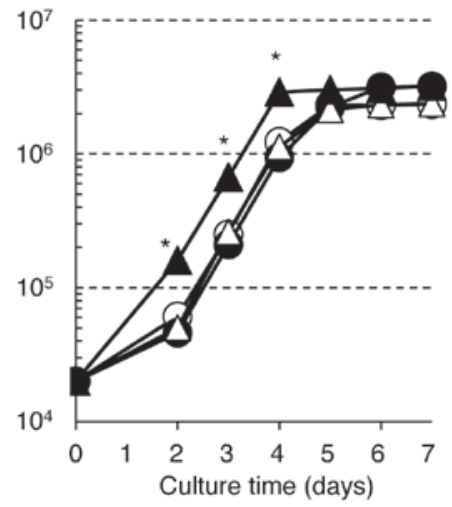

$\mathbf{E}$

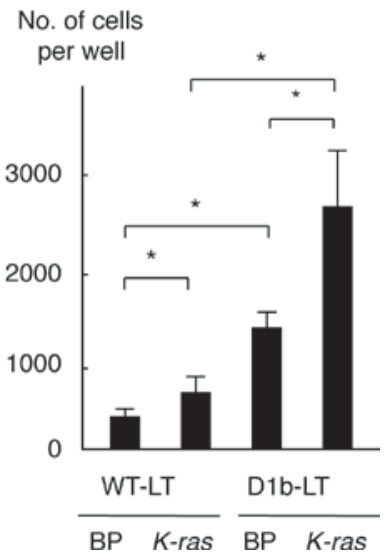

Figure 1. Cooperation of cyclin D1b with activated $K$-ras in the transformation and tumorigenicity of mouse embryo fibroblast cells. (A) Morphology of MEF cells expressing cyclin D1b and/or activated $K$-ras. Magnification, x100. (B) Growth curves of MEF cells expressing cyclin D1b and/or activated $K$-ras. White circle, black circle, white triangle and black triangle represent WT-LT/BP, WT-LT/K-ras, D1b-LT/BP and Dlb-LT/K-ras cells, respectively. Each sample was assayed in triplicate, and the bar represents the mean $\pm \mathrm{SD}$. " $\mathrm{P} \leq 0.05$, for D1b-LT/K-ras cells compared with the remaining cell lines at days 2,3 and 4 . (C) Anchorage-independent growth of MEF cells expressing cyclin D1b and/or activated $K$-ras. Each sample was assayed in triplicate, and the bar represents the mean $\pm \mathrm{SD}$. ${ }^{* *} \mathrm{P}<0.01$. (D) Tumorigenicity in nude mice of MEF cells expressing cyclin D1b and/or activated $K$-ras. Each sample was assayed in triplicate, and the bar represents the mean $\pm \mathrm{SD} .{ }^{*} \mathrm{P}=0.039$. (E) Invasiveness of MEF cells expressing cyclin D1b and/or activated $K$-ras. Each sample was assayed in triplicate, and the bar represents the mean $\pm \mathrm{SD}$. ${ }^{*} \mathrm{P}<0.05$. K-ras, v-K-ras2 Kirsten rat sarcoma viral oncogene homolog; MEF, mouse embryo fibroblast; WT, wild-type; SD, standard deviation; BP, pBABE puro.

A

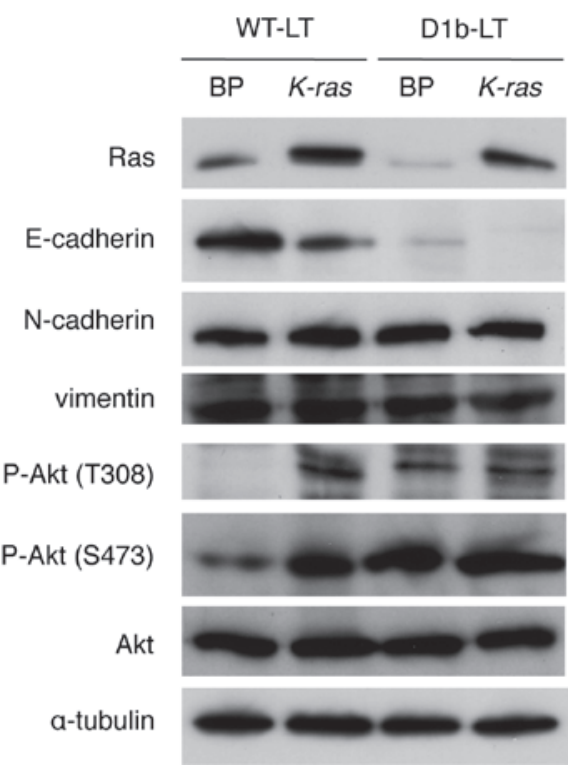

B No. of cells per well

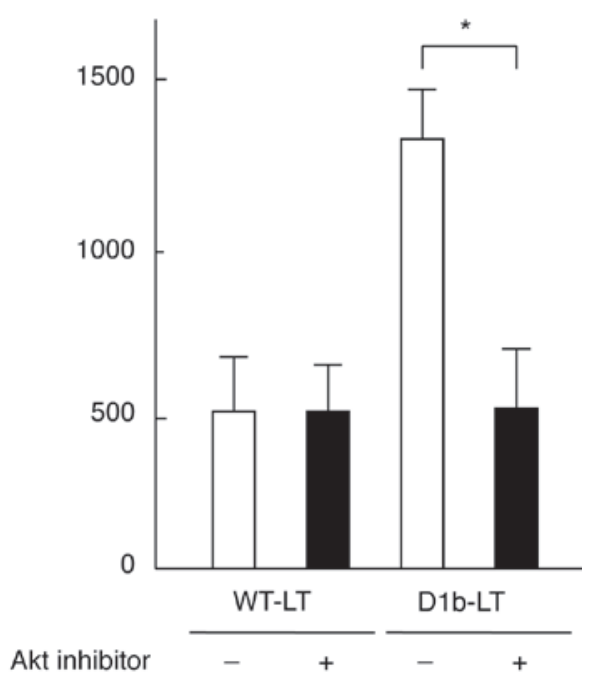

Figure 2. Alterations in expression of proteins involved in the EMT and phosphorylation of Akt in MEF cells expressing cyclin Dlb and/or activated $K$-ras. (A) Immunoblot analyses of the expression of EMT proteins and the phosphorylation of Akt. (B) Effect of an Akt inhibitor on the invasiveness of MEF cells expressing cyclin Dlb. The Akt inhibitor was used at a concentration of $25 \mu \mathrm{M}$. Each sample was assayed in triplicate, and the bar represents the mean \pm standard deviation. $\mathrm{P}=0.9947$, compared with untreated WT-LT MEF cells. " $\mathrm{P}=0.0116$. EMT, epithelial-to-mesenchymal transition; MEF, mouse embryo fibroblast; K-ras, v-K-ras2 Kirsten rat sarcoma viral oncogene homolog; WT, wild-type; P, phosphorylated; BP, pBABE puro. 
A

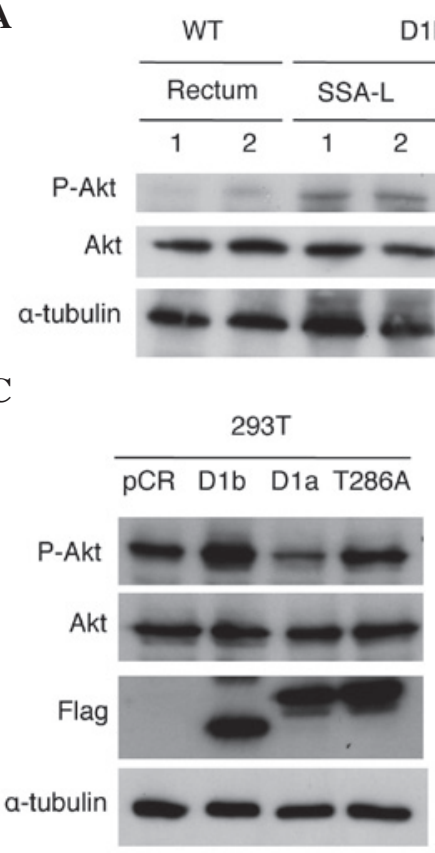

B

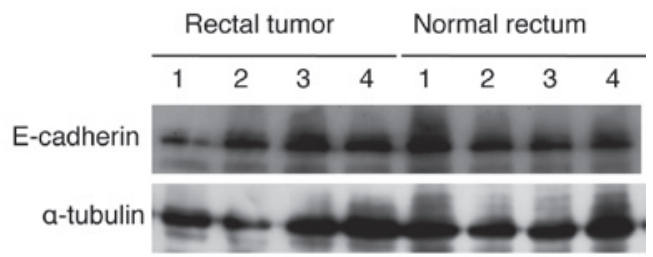

E

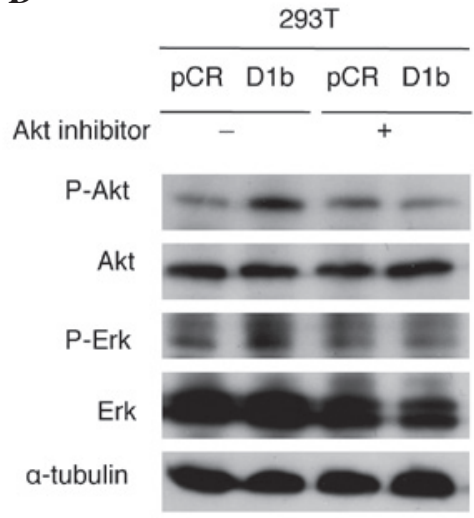

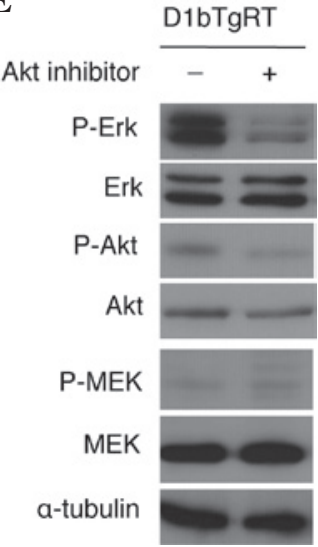

Figure 3. Involvement of Akt in Erk activation by cyclin D1b in the rectal tumors of the cyclin D1b Tg mouse, the D1bTgRT tumor cell line and human 293 cells. (A) Immunoblot analysis of Akt activation in normal rectal tissue from female WT mice and rectal tumors with or without adenocarcinomas from cyclin D1b Tg mice. $\alpha$-tubulin was used as an internal control. (B) Immunoblot analysis of E-cadherin expression in normal rectal tissue from female WT mice and rectal tumors from cyclin D1b Tg mice. $\alpha$-tubulin was used as an internal control. (C) Immunoblot analysis of Akt activation in $293 \mathrm{~T}$ cells transfected with cyclin D1b, cyclin D1a and cyclin D1-T286A. $\alpha$-tubulin was used as an internal control. (D) Effect of an Akt inhibitor on Erk activation by cyclin D1b in $293 \mathrm{~T}$ cells. The Akt inhibitor was at a concentration of $25 \mu \mathrm{M}$ for $24 \mathrm{~h}$. $\alpha$-tubulin was used as an internal control. (E) Effect of an Akt inhibitor on Erk activation by cyclin D1b in D1bTgRT cells. The Akt inhibitor was used at a concentration of $25 \mu \mathrm{M}$ for $24 \mathrm{~h}$. $\alpha$-Tubulin was used as an internal control. Erk, extracellular signal-regulated kinase; WT, wild-type; SSA-L, sessile serrated adenoma-like lesion; AC, adenocarcinoma; MEK, mitogen-activated protein kinase/Erk kinase.

residues) was enhanced in WT-LT/K-ras, D1b-LT/BP and $\mathrm{D} 1 \mathrm{~b} / K$-ras cells when compared with WT-LT/BP cells. The levels of total Akt and $\alpha$-tubulin protein were similar in all cell lines. These results indicate that cyclin D1b is able to suppress E-cadherin expression and to activate Akt in MEF cells. To clarify whether Akt activation is necessary for an increase of cell invasiveness by cyclin D1b, a Matrigel invasion assay of WT-LT and D1b-LT MEF cells treated with an Akt inhibitor $(25 \mu \mathrm{M})$ was performed. As shown in Fig. 2B, the Akt inhibitor significantly suppressed the invasiveness of D1b-LT MEF cells $(\mathrm{P}=0.0116)$, but not of WT-LT MEF cells $(\mathrm{P}=0.9947)$, indicating that Akt activation has a significant role for the enhancement of cell invasiveness in cyclin Dlb-expressing MEF cells.

Akt activation in rectal tumors of cyclin Dlb $\mathrm{Tg}$ mice and cyclin Dlb-expressing 2937 cells. To clarify the role of Akt in the rectal tumorigenicity of cyclin D1b Tg mice, the present study examined the phosphorylation of Akt in normal rectum and rectal tumors (SSA/Ps and adenocarcinomas) of cyclin D1b Tg mice. As shown in Fig. 3A, phosphorylation of Akt was increased in rectal tumors as compared with normal rectal tissue. As previously reported, Erk was also activated in rectal tumors without activation of MEK (14). Although the present study also examined the expression of E-cadherin in normal rectum and rectal tumors, alteration of its expression was not observed in the rectal tumors of these $\mathrm{Tg}$ mice
(Fig. 3B). The present study also investigated Akt activation in 293T cells transfected with cyclin D1b, cyclin D1a and cyclin D1-T286A. Cyclin D1-T286A is a mutant of cyclin D1a, in which threonine-286 (T286), the site of glycogen synthase kinase- $3 \beta$ phosphorylation that is required to enhance both the nuclear export of cyclin D1 and its turnover, is replaced with alanine (22). The mutation of T286 to a nonphosphorylatable residue promotes a constitutively nuclear localization of the cyclin Dla protein, with enhanced oncogenic potential (23). As shown in Fig. 3C, increased expression of cyclin D1b and cyclin D1-T286A by transfection accelerated the phosphorylation of Akt, suggesting that nuclear localization of these cyclin D1 proteins is critical for Akt activation. The present authors have previously reported that expression of cyclin D1b increases Erk phosphorylation without activating MEK in 293T cells (14). To clarify whether activation of Erk by cyclin D1b is mediated through Akt activation, the present study investigated the effects of an Akt inhibitor on Erk activation by cyclin D1b in 293T cells. As shown in Fig. 3D, treatment with an Akt inhibitor suppressed the enhanced phosphorylation of Erk and Akt in 293T cells expressing cyclin D1b. The present study also investigated the effects of the Akt inhibitor on Erk activity in D1bTgRT cells from a rectal tumor of the cyclin D1b Tg mouse. As shown in Fig. 3E, treatment of the Akt inhibitor suppressed phosphorylation of Erk and Akt in D1bTgRT cells. These results show that cyclin D1b activates Erk via Akt to contribute to tumor formation. 
A

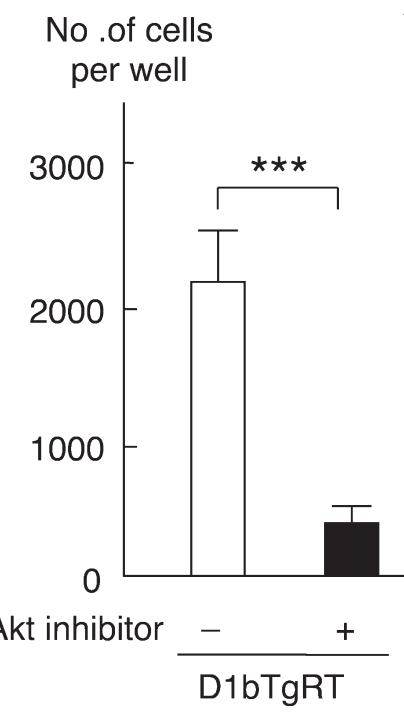

B

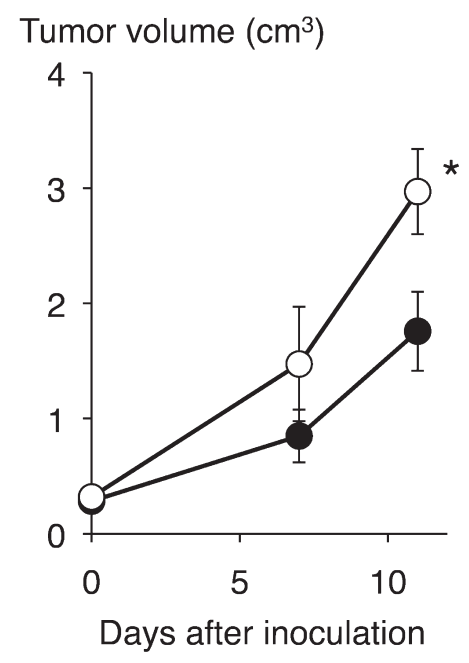

Figure 4. Effect of an Akt inhibitor on cell invasiveness and tumor formation in nude mice injected with DlbTgRT cells. (A) Matrigel invasion assay. Each sample was assayed in triplicate, and the bar represents the mean $\pm \mathrm{SD}$. ${ }^{* * *} \mathrm{P}=0.0007$. The Akt inhibitor was used at a concentration of $25 \mu \mathrm{M}$. (B) Tumorigenicity assay. Each sample was assayed in triplicate, and the bar represents the mean \pm SD. ${ }^{*} \mathrm{P}=0.0425$. SD, standard deviation.

Effect of Akt inhibitor on cell invasiveness and tumorigenicity of DlbTgRT cells. To clarify the role of Akt in the cell invasiveness of rectal tumor cells expressing cyclin D1b, the present study examined the effect of an Akt inhibitor on the cell invasiveness of D1bTgRT cells. As shown in Fig. 4A, treatment with the Akt inhibitor $(25 \mu \mathrm{M})$ significantly reduced the cell invasiveness of D1bTgRT cells $(\mathrm{P}=0.0007)$. Furthermore, the present study examined the effect of Akt inhibition on tumor growth in nude mice. As shown in Fig. 4B, the tumor growth of D1bTgRT cells was significantly decreased by Akt inhibitor treatments $(\mathrm{P}=0.0425)$. These results indicate that enhanced phosphorylation of Akt by cyclin Dlb contributes to cell invasiveness and tumor formation of the rectal tumor cell line, D1bTgRT.

\section{Discussion}

In the present study, it was initially demonstrated that cyclin D1b has the ability to enhance cell invasiveness by itself; it is also able to increase cell invasiveness, anchorage-independent growth and tumorigenicity in cooperation with an activated $K$-ras oncogene in MEF cells. These results are consistent with the in vivo activity of cyclin D1b that leads to rectal tumorigenesis in the cyclin D1b-Tg mouse (14). The present study also observed that phosphorylation of Akt increased in cyclin D1b-expressing MEF cells. Phosphorylation of Akt was also enhanced in the rectal tumor tissues of the cyclin D1b Tg mice. Furthermore, Akt was also activated by transfection of cyclin D1b, but not cyclin Dla, in human $293 \mathrm{~T}$ cells. These results suggest that Akt activation is correlated with Erk activation, as previously described (14). Treatment with an Akt inhibitor suppresses activation of Erk in 293T cells expressing cyclin D1b. Furthermore, the Akt inhibitor also suppresses activation of Erk and the cell invasiveness and tumorigenicity of DlbTgRT cells derived from a rectal tumor of the cyclin Dlb Tg mouse. These results show that cyclin Dlb activates Erk via Akt and suggest that activation of Akt contributes to the tumorigenicity induced by cyclin Dlb.

The present authors previously constructed cyclin D1b $\mathrm{Tg}$ mice and observed that rectal tumors, including SSA and adenocarcinoma, were generated at a high frequency $(60 \%)$ in the female $\mathrm{Tg}$ mice (14). It was additionally observed that the phosphorylation of Erk was enhanced without activation of MEK and hotspot mutations of $K$-ras and $B$-raf in the rectal tumors from the Tg mice and in MEF cells expressing cyclin D1b (14). siRNA-induced cyclin D1b knockdown in DlbTgRT cells reduced phosphorylation of Erk and the malignant phenotypes, including anchorage-independent growth, cell invasiveness and tumorigenicity, indicating that Erk activation by cyclin D1b is critical for tumor formation (14). Furthermore, the transient expression of cyclin D1b enhanced phosphorylation of Erk without activation of MEK in $293 \mathrm{~T}$ cells (14). However, in this previous study, it was not possibly to clarify how cyclin D1b activates Erk without the activation of upstream signal pathway elements, including MEK, B-Raf and K-Ras (14). As activation of Akt by cyclin Dlb was observed in MEF cells, the present study focused on the role of Akt in Erk activation via a MEK-independent signaling pathway by cyclin D1b and demonstrated this in rectal tumors of cyclin Dlb Tg mice, in a tumor cell line, DlbTgRT, derived from a rectal tumor in a $\mathrm{Tg}$ mouse, and in cyclin Dlb-expressing 293 T cells. Previously, Aksamitiene et al (24) have demonstrated that phosphoinositide 3-kinase (PI3K)/Akt activates Erk via T-lymphokine-activated killer cell-originated protein kinase or an unidentified kinase downstream of the EGF receptor in breast cancer. Akt is known to associate with a variety of substrate and nonsubstrate proteins that regulate its kinase activity (25). It is possible that cyclin Dlb binds to Akt or one of its regulating molecules to regulate Akt activity. Although the present authors attempted to detect the binding of Akt, PI3K and 3-phosphoinositide dependent protein kinase-1 with cyclin D1b in 293T cells expressing cyclin D1b, it was not possible to demonstrate the binding of these proteins 
under the present experimental conditions. As cyclin D1b protein is predominantly localized in the nucleus, this protein may activate the PI3K/Akt signaling pathway in an indirect manner through transcriptional regulation of Akt-activating proteins. A constitutively nuclear cyclin D1a mutant protein, cyclin D1-T286A, also accelerates phosphorylation of Akt, supporting this idea. Through whichever mechanism, Akt is considered to have a significant role in Erk activation and the tumorigenicity of cyclin D1b, although further investigation is required for the identification of the direct target protein(s) of cyclin D1b and the clarification of the molecular mechanism(s) of Akt activation by cyclin D1b.

In addition to the Erk signaling pathway, which is predominantly involved in regulation of cell proliferation, PI3K/Akt signaling has diverse functions, including suppression of apoptosis, cell survival, cell growth and regulation of cell metabolism, and has been shown to have a role in the progression of cancer (26-28). In numerous types of human cancer, including bladder cancer, esophageal cancer, colorectal cancer, B-lymphoid malignancies, breast cancer and prostate cancer (4), all of which have been shown to express the cyclin Dlb variant, inhibitors targeting this signaling pathway are expected to have therapeutic potential.

\section{Acknowledgements}

The authors would like to thank Mrs. Akiyo Ushio (Division of Microbiology and Infectious Diseases, Shiga University of Medical Science, Otsu, Japan) for her technical assistance with experiments. The study was supported by the Japan Society for the Promotion of Science KAKENHI (Grants-in-aid for Scientific Research) (grant nos. 19591843 and 24590480).

\section{References}

1. Knudsen KE, Diehl JA, Haiman CA and Knudsen ES: Cyclin D1: Polymorphism, aberrant splicing and cancer risk. Oncogene 25: 1620-1628, 2006.

2. Sherr CJ: D-type cyclins. Trends Biochem Sci 20: 187-190, 1995.

3. Lukas J, Parry D, Aagaard L, Mann DJ, Bartkova J, Strauss M, Peters G and Bartek J: Retinoblastoma-protein-dependent cell-cycle inhibition by tumour suppressor p16. Nature 375: 503-506, 1995 .

4. Musgrove EA, Caldon CE, Barraclough J, Stone A and Sutherland RL: Cyclin D as a therapeutic target in cancer. Nat Rev Cancer 11: 558-572, 2011.

5. Hosokawa Y, Gadd M, Smith AP, Koerner FC, Schmidt EV and Arnold A: Cyclin D1 (PRAD1) alternative transcript b: Full-length cDNA cloning and expression in breast cancers. Cancer Lett 113: 123-130, 1997.

6. Lu F, Gladden AB and Diehl JA: An alternatively spliced cyclin D1 isoform, cyclin D1b, is a nuclear oncogene. Cancer Res 63 : 7056-7061, 2003.

7. Solomon DA, Wang Y, Fox SR, Lambeck TC, Giesting S, Lan Z, Senderowicz AM, Conti CJ and Knudsen ES: Cyclin D1 splice variants. Differential effects on localization, RB phosphorylation, and cellular transformation. J Biol Chem 278: 30339-30347, 2003.

8. Burd CJ, Petre CE, Morey LM, Wang Y, Revelo MP, Haiman CA, Lu S, Fenoglio-Preiser CM, Li J, Knudsen ES, et al: Cyclin D1b variant influences prostate cancer growth through aberrant androgen receptor regulation. Proc Natl Acad Sci USA 103: 2190-2195, 2006.
9. Holley SL, Parkes G, Matthias C, Bockmühl U, Jahnke V, Leder K, Strange RC, Fryer AA and Hoban PR: Cyclin D1 polymorphism and expression in patients with squamous cell carcinoma of the head and neck. Am J Pathol 159: 1917-1924, 2001.

10. Howe D and Lynas C: The cyclin D1 alternative transcripts[a] and [b] are expressed in normal and malignant lymphocytes and their relative levels are influenced by the polymorphism at codon 241. Haematologica 86: 563-569, 2001.

11. Bala S and Peltomäki P: Cyclin D1 as a genetic modifier in hereditary nonpolyposis colorectal cancer. Cancer Res 61: 6042-6045, 2001.

12. Paronetto MP, Cappellari M, Busà R, Pedrotti S, Vitali R, Comstock C, Hyslop T, Knudsen KE and Sette C: Alternative splicing of the cyclin D1 proto-oncogene is regulated by the RNA-binding protein Sam68. Cancer Res 70: 229-239, 2010.

13. Kim CJ, Nishi K, Isono T, Okuyama Y, Tambe Y, Okada Y and Inoue $\mathrm{H}$ : Cyclin D1b variant promotes cell invasiveness independent of binding to CDK4 in human bladder cancer cells. Mol Carcinog 48: 953-964, 2009.

14. Kim CJ, Tambe Y, Munkaisho K, Sugihara H, Isono T, Sonoda H, Shimizu T, Kondoh G and Inoue H: Female-specific rectal carcinogenesis in cyclin D1b transgenic mice. Carcinogenesis 35: 227-236, 2014.

15. Snover DC, Ahnen D, Burt R and Odze RD: Serrated polyps of the colon and rectum and serrated polyposis. In: WHO classification of tumors of the digestive system. Bozman FT, Carneiro F, Hruban RH and Theise ND (eds.) 4th edition. Springer-Verlag, Berlin, Germany, pp 160-165, 2010.

16. Snover DC, Jass JR, Fenoglio-Preiser C and Batts KP: Serrated polyps of the large intestine: A morphologic and molecular review of an evolving concept. Am J Clin Pathol 124: 380-391, 2005.

17. East JE, Saunders BP and Jass JR: Sporadic and syndromic hyperplastic polyps and serrated adenomas of the colon: Classification, molecular genetics, natural history, and clinical management. Gastroenterol Clin North Am 37: 25-46, 2008.

18. Leggett $B$ and Whitehall V: Role of the serrated pathway in colorectal cancer pathogenesis. Gastroenterology 138: 2088-2100, 2010.

19. Snover DC: Update on the serrated pathway to colorectal carcinoma. Hum Pathol 42: 1-10, 2011.

20. Rex DK, Ahnen DJ, Baron JA, Batts KP, Burke CA, Burt RW, Goldblum JR, Guillem JG, Kahi CJ, Kalady MF, et al: Serrated lesions of the colorectum: Review and recommendations from an expert panel. Am J Gastroenterol 107: 1315-1329; quiz 1314, 1330, 2012.

21. Tambe Y, Hasebe M, Kim CJ, Yamamoto A and Inoue H: The drs tumor suppressor regulates glucose metabolism via lactate dehydrogenase-B. Mol Carcinog 55: 52-63, 2016.

22. Alt JR, Cleveland JL, Hannink M and Diehl JA: Phosphorylation-dependent regulation of cyclin D1 nuclear export and cyclin D1-dependent cellular transformation. Genes Dev 14: 3102-3114, 2000.

23. Diehl JA, Cheng M, Roussel MF and Sherr CJ: Glycogen synthase kinase-3beta regulates cyclin D1 proteolysis and subcellular localization. Genes Dev 12: 3499-3511, 1998.

24. Aksamitiene E, Kholodenko BN, Kolch W, Hoek JB and Kiyatkin A: PI3K/Akt-sensitive MEK-independent compensatory circuit of ERK activation in ER-positive PI3K-mutant T47D breast cancer cells. Cell Signal 22: 1369-1378, 2010.

25. Brazil DP, Park J and Hemming BA: PKB binding proteins. Getting in on the Akt. Cell 111: 293-303, 2002.

26. Vivanco I and Sawyers CL: The phosphatidylinositol 3-kinase-Akt pathway in human cancer. Nat Rev Cancer 2: 489-501, 2002.

27. Downward J: Targeting Ras signaling pathways in cancer therapy. Nat Rev Cancer 3: 11-22, 2003.

28. Engelman JA: Targeting PI3K signaling in cancer: Opportunities, challenges and limitations. Nat Rev Cancer 9: 550-562, 2009. 